\title{
Optimum Axillary Dissection in Breast Cancer-is Angular Vein a Reliable Spatial Landmark?
}

\author{
Chintamani
}

Published online: 18 March 2011

(C) Indian Association of Surgical Oncology 2011

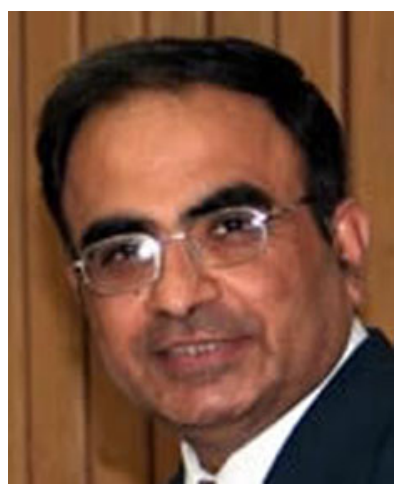

Chintamani

Axillary lymph nodes are the surrogate markers of outcome in patients with breast cancer. The survival drops by nearly $50 \%$ in patients with positive axillae. The "gold standard" method of assessing the axilla is to establish the histological evidence of disease in the nodes, making assessment of axilla mandatory even in node negative patients [1-3].

With the change in the attitude of most cancer surgeons towards organ and function preservation, there has been a paradigm shift in the intent of the surgeon towards being more and more conservative. In node negative axillae, sentinel lymph node biopsy (SLNB) and /or four node axillary sampling is performed to ascertain its status, while a conventional axillary dissection is mandatory in patients with positive axillae. Inspite of a change in the attitude towards being more and more conservative, oncological safety remains of paramount importance and addressing

\section{Chintamani $(\bowtie)$}

VMMC, Safdarjang Hospital,

New Delhi, India

e-mail: chintamani7@rediffmail.com axilla is mandatory. Regarding various anatomical end points for optimum axillary dissection there are no definite guidelines and optimum axillary dissection is often a subject of controversy.

Some surgeons consider clearance of levels-I and II to be optimum while most surgeons would like to dissect up to level-III. It is often debated that clearing level-III may actually add to the morbidity of axillary vein thrombosis and lymphaedema without rendering any survival advantage. The counter argument to this thought is related to the inadequacy of dissection defeating the therapeutic intent of axillary clearance. The morbidity of axillary clearance up to level-III is minimal at the hands of experienced surgeons at a

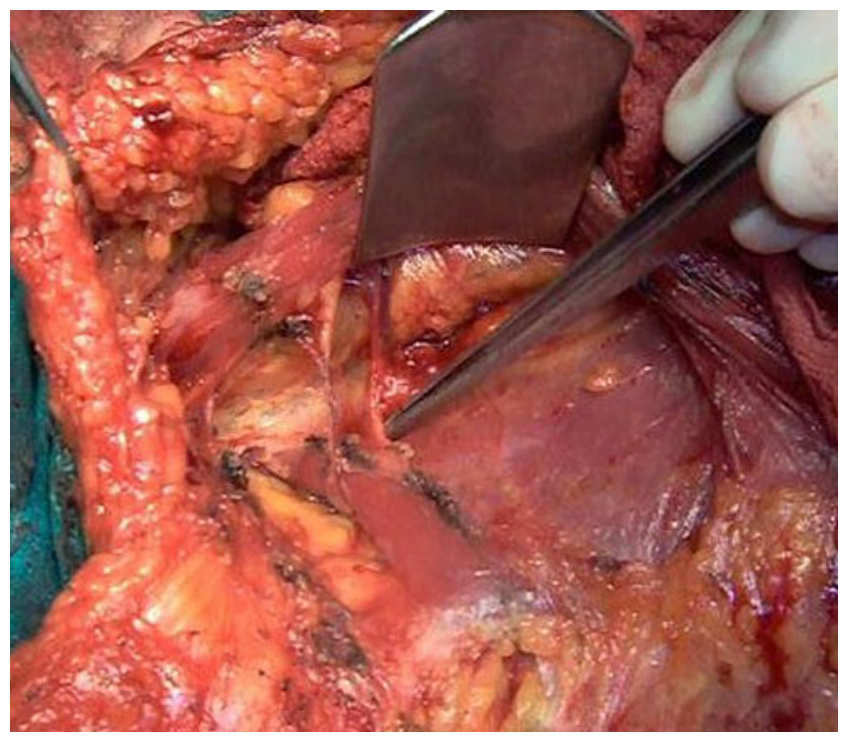

Fig. 1 Axillary dissection in progress in a patient with locally advanced breast cancer. The medial and lateral pectoral nerves may be seen. The medial pectoral nerve is lateral in location. Preserving these nerves is essential if the pectoral muscles are preserved like in the presented case 


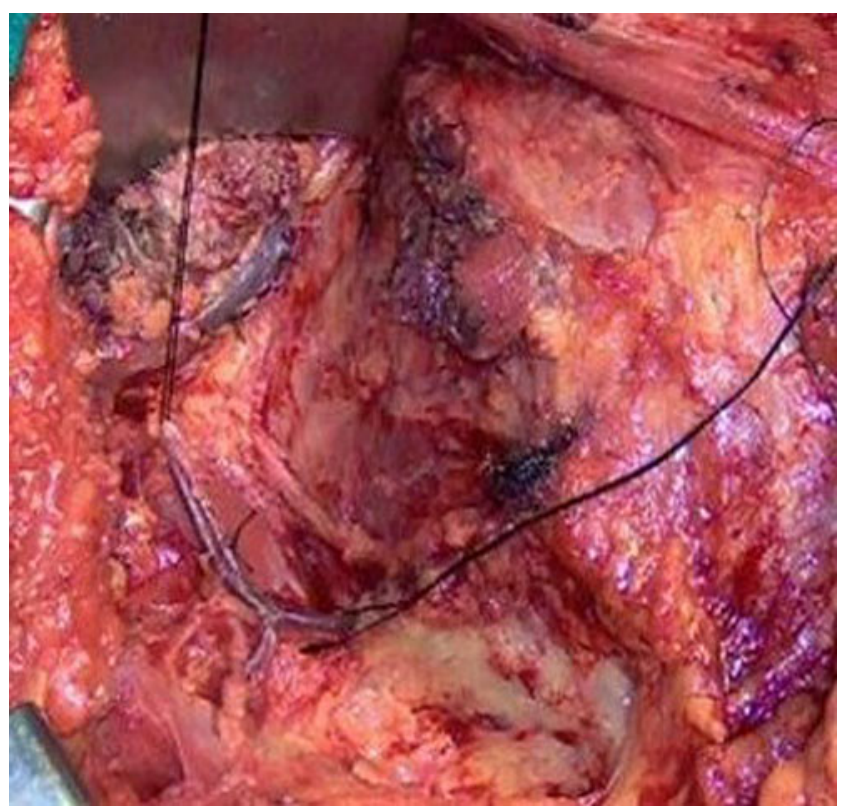

Fig. 2 The angular vein (the lower silk thread marker) is seen forming the lower limit of axillary dissection, while the lateral limit is formed by the thoraco-dorsal pedicle (upper silk thread marker) and tendon of lattismus dorsii muscle. The axillary vein forms the superior limit of axillary dissection. The triangle shown has to be cleared of all the lymphatics while the nerve to serratus anterior and thoracodorsal nerve should be preserved as shown

high volume center and is recommended by most surgeons including the author.

We routinely like to clear all the three levels and have found minimal morbidity if adequate precautions are taken during axillary dissections(avoiding electrocautery close to the vein) and following it up with half or low suction drainage [1]. This technique ensures a satisfactory axillary clearance; reduced morbidity of seroma formation and delayed effects like lymphadema [1, 3]. It is generally accepted that the optimum axillary dissection should yield at least ten lymph nodes for accurate prognostication [3].

Regarding the anatomical end points that should be met during axillary dissections, most surgeons consider the medial limit of dissection to be costo-clavicular ligament also known as 'Halstead's ligament' while laterally the tendon of Lattismus Dorsi(LD) and thoracodorsal pedicle (containing thoracodorsal nerve, artery and subscapular vein) are the reliable anatomical end points. The superior limit of axillary dissection is considered to be axillary vein while the floor is formed by subscapularis muscle (Figs. 1 and 2). Regarding the lower limit of axillary dissection, "angular vein" (that joins thoracodorsal vein to form the subscapular vein) has been found by many authors to be a reliable and constant landmark [2]. We have also observed it to be a reliable anatomical end point for the lower limit of dissection in nearly four hundred axillae that were dissected for breast cancer. In a classical axillary lymph node dissection, the triangular space (bound by axillary vein superiorly, thoracodorsal pedicle and tendon of LD leterally, Halstead ligament medially and angular vein inferiorly) has to be cleared of all lympho-fatty tissue preserving thoracodorsal nerve, nerve to serratus anterior and the pectoral nerves (medial and lateral).

It is important to realize that preservation of these nerves is mandatory along side preservation of pectoral muscles. In real life situation, the medial pectoral nerve is lateral; it is called so because of the cord of brachial plexus from which it originates (Figs. 1 and 2).

The author ensures that these landmarks are dissected and exposed each time the axilla is dissected for optimum yield. We have found these landmarks to be very reliable in ensuring an optimum axillary dissection in more than four hundred cases. The average number of lymph nodes dissected in this series was more than 10 with a mean of 14 nodes [1]. It is essential for every breast surgeon to ensure that axillary dissection is optimum and these landmarks can help in this effort by providing the anatomical end points to be achieved each time the axilla is dissected. Angular vein can be reliably used as an antomical landmark for lower limit of axillary dissection

\section{References}

1. Chintamani, Singhal V, Singh J, Bansal A, Saxena S (2005) Half versus full vacuum suction drainage after modified radical mastectomy for breast cancer-a prospective randomized clinical trial[ISRCTN24484328]. BMC Cancer 5(1):11

2. Chan CY, Tan M (2003) Spatial relations of the angular vein, an important landmark in maxillary nodal dissection. Br J Surg 8:948949

3. Benson JR, Quirky Della Revere G (2007) Management of the maxilla in women with breast cancer. Breast 16(2):130-136 